CLINICAL STUDY

\title{
Acute changes in serum osteoprotegerin and receptor activator for nuclear factor- $\kappa B$ ligand levels in women with established osteoporosis treated with teriparatide
}

\author{
Athanasios D Anastasilakis, Dimirtios G Goulis ${ }^{1}$, Stergios A Polyzos, Spiridon Gerou ${ }^{2}$, Vasiliki Pavlidou ${ }^{2}$, \\ George Koukoulis ${ }^{3}$ and Avraam Avramidis \\ Department of Endocrinology, Hippocration General Hospital, 54642 Thessaloniki, Greece, ${ }^{1}$ Unit of Reproductive Endocrinology, First Department of \\ Obstetrics and Gynecology, Aristotle University of Thessaloniki, 54603 Thessaloniki, Greece, ${ }^{2}$ Analysis Laboratories, 54623 Thessaloniki, Greece and \\ ${ }^{3}$ Department of Internal Medicine, University of Thessalia, 41222 Larissa, Greece \\ (Correspondence should be addressed to A D Anastasilakis who is now at Soulini 4, 56625 Sykies, Greece; Email: anastath@endo.gr)
}

\begin{abstract}
Objective: The mechanisms regulating the anabolic response of the skeleton to intermittent exogenous parathyroid hormone (PTH) administration are not fully elucidated. The aim of this prospective study was to evaluate the acute effect (up to 1 month) of teriparatide (TPTD; human recombinant PTH 1-34) on serum levels of osteoprotegerin (OPG) and receptor activator for nuclear factor- $\kappa \mathrm{B}$ ligand (RANKL) in women with established osteoporosis.

Design: Twenty-three postmenopausal Caucasian women with established osteoporosis (mean age $66.7 \pm 1.6$ years) received daily injections of $20 \mu \mathrm{g}$ TPTD for 12 months.

Methods: Serum samples for total calcium (Ca), phosphate, alkaline phosphatase, N-terminal propeptide of type I collagen, intact PTH (iPTH), OPG, and RANKL were obtained at baseline, $1 \mathrm{~h}$, 1 day, and 1 month after initiation of therapy. Lumbar spine bone mineral density (BMD) was measured before and 12 months after TPTD treatment.

Results: Serum total Ca increased and iPTH gradually decreased with TPTD treatment. Serum OPG levels remained unchanged, while RANKL increased gradually during the study $(P<0.001)$. There was no correlation between OPG or RANKL and BMD changes or iPTH levels.

Conclusions: TPTD therapy in women with postmenopausal osteoporosis results in acute increase in serum RANKL levels but does not affect serum OPG. These changes may reflect an increase in the number of active osteoblasts with therapy and might be responsible for the acceleration of bone turnover rate that characterizes TPTD.
\end{abstract}

European Journal of Endocrinology 158 411-415

\section{Introduction}

Parathyroid hormone (PTH) exerts a catabolic effect on the skeleton in the case of continuous hypersecretion, such as in primary hyperparathyroidism. On the contrary, intermittent administration of teriparatide (TPTD; recombinant human PTH 1-34) exerts an osteoanabolic effect characterized by direct effects on bone formation through stimulation of osteoblast activity and inhibition of their apoptosis (1). Nevertheless, the mechanisms regulating the anabolic response of TPTD to the skeleton are not fully elucidated.

The system of osteoprotegerin (OPG), receptor activator for nuclear factor- $\mathrm{KB}$ (RANK) and its ligand (RANKL) plays a central role in the pathophysiology of osteoporosis. RANKL, expressed by the pre-osteoblasts and stromal cells, binds to RANK, expressed by cells of the osteoclast lineage, inducing a signaling cascade leading to the differentiation and fusion of osteoclast precursor cells and stimulating the activity of the mature osteoclast (2). The effects of RANKL are counteracted by OPG, a soluble neutralizing decoy receptor. OPG and RANKL production by the osteoblasts is regulated by a number of cytokines and hormones, including PTH (3). RANKL is expressed preferably by undifferentiated osteoblasts. As the osteoblasts differentiate, RANKL mRNA levels decrease and OPG levels increase (2). Therapies that modify bone turnover are expected to influence the production of OPG and RANKL.

In vitro studies in both animal and human osteoblasts indicate that PTH decreases (4-6) or does not affect OPG (7) and increases RANKL production (4-6). Intracellular calcium $(\mathrm{Ca})$ elevations seem to mediate these actions on OPG/RANKL expression (8). In vivo animal studies showed that PTH, delivered either intermittently $(9,10)$ or continuously $(11,12)$, leads to increased expression of RANKL gene, while OPG expression seems 
not to be altered (12). Similarly, in patients with hyperparathyroidism, bone biopsies showed an increase in RANKL and a decrease in OPG gene expression, which was reversed after parathyroidectomy (13), although serum OPG did not correlate with PTH before surgery and was not affected by parathyroidectomy (14). In another study, endogenous PTH levels were negatively associated with OPG expression, but also, surprisingly, with RANKL expression in human bone tissue in vivo (15). Only one study has examined the long-term effects of TPTD on serum OPG and RANKL levels in humans with glucocorticoid-induced osteoporosis (16). In this study, Buxton et al. reported an early-onset significant increase in serum RANKL, followed by a mild decrease in OPG. To the best of our knowledge, no study has reported on the acute impact of TPTD in OPG/RANKL serum levels.

The aim of this prospective study was to evaluate the acute effect of TPTD administration on serum OPG and RANKL levels, in women with established osteoporosis.

\section{Patients and methods}

\section{Patients}

Patients were recruited at the outpatient clinics of the Department of Endocrinology, Hippocration General Hospital, Thessaloniki, Greece, a tertiary referral center for metabolic bone diseases. Twenty-three consecutive postmenopausal Caucasian women (age 66.7 \pm 1.6 years, age at menopause $45.2 \pm 1.0$ years) with established osteoporosis were included. Established osteoporosis was defined as a T-score of less than -2.5 (mean $-3.1 \pm 0.2$ ) at the lumbar spine and the presence of at least one vertebral or non-vertebral fracture (mean 1.4 \pm 0.1 fractures). Exclusion criteria were: i) secondary osteoporosis, ii) diseases that could affect bone metabolism, and iii) medications that could affect bone metabolism. Women receiving any antiosteoporotic treatment during the last 6 months were excluded as well. All patients were receiving $500 \mathrm{mg}$ elemental Ca and 400 IU vitamin D daily throughout the study. Patients' baseline characteristics are given in Table 1. This study was approved by the Ethics Committee of Hippocration General Hospital.

\section{Methods}

Baseline assessment included history, clinical evaluation, and body mass index (BMI). Morning fasting blood samples were obtained from all women before (baseline), $1 \mathrm{~h}, 1$ day, and 1 month after initiation of treatment. The samples were centrifuged immediately and serum was separated and stored at $-30{ }^{\circ} \mathrm{C}$; all measurements were performed simultaneously at the end of the study. Studied parameters included serum levels of total $\mathrm{Ca}$, phosphate $(\mathrm{P})$, total alkaline
Table 1 Patient characteristics at baseline.

\begin{tabular}{lccc}
\hline & Mean \pm s.E.M. & Min & Max \\
\hline Age (years) & $66.7 \pm 1.6$ & 53 & 77 \\
Menopausal age (years) & $45.2 \pm 1.0$ & 38 & 52 \\
Body weight (kg) & $66.9 \pm 2.5$ & 50 & 92 \\
BMl (kg/m ${ }^{2}$ ) & $28.4 \pm 1.0$ & 21.6 & 39.5 \\
BMD at lumbar spine & $0.758 \pm 0.034$ & 0.623 & 0.872 \\
$\quad\left(g / \mathrm{cm}^{2}\right)$ & $1.4 \pm 0.1$ & 1 & \\
Number of fractures & $1.1 \pm 0.1$ & 0 & 4 \\
$\quad$ At vertebral column & $0.1 \pm 0.1$ & 0 & 1 \\
$\quad$ At hip & $0.2 \pm 0.1$ & 0 & 1 \\
$\quad$ At other site &
\end{tabular}

BMI, body mass index, calculated as body weight $(\mathrm{kg}) /$ height $(\mathrm{m})^{2}$; BMD, bone mineral density.

phosphatase (ALP; COBAS INTEGRA ALP IFCC; Roche Diagnostics), intact PTH (iPTH; ECLIA, Elecsys PTH; Roche Diagnostics), OPG (ELISA; Ray Biotech Inc., Nocross, Georgia, USA; sensitivity $0.02 \mathrm{pmol} / \mathrm{l}$, intra-assay coefficient of variation $(\mathrm{CV})<10 \%$, interassay CV $<12 \%$ ), and RANKL (ELISA; Biomedica, Wien, Austria; sensitivity $0.08 \mathrm{pmol} / \mathrm{l}$, intra-assay CV $<3-5 \%$, inter-assay $\mathrm{CV}<6-9 \%$ ). The OPG assay detects all three different forms of circulating OPG (monomer, dimer, and RANKL/OPG complex). Serum N-terminal propeptide of type 1 collagen (P1NP; ECLIA, Elecsys total P1NP; Roche Diagnostics), a more specific bone formation marker, was also measured at baseline and 1 month in order to verify TPTD effects and compliance.

Lumbar spine bone mineral density (BMD) was measured by dual energy X-ray absorptiometry using a DPX-IQ densitometer (Lunar Corporation, Madison, WI, USA) before and 12 months after initiation of treatment.

\section{Statistical analysis}

Based on a pilot study of our group, regarding baseline and post-treatment levels of study variables, we assumed the following a priori levels: serum RANKL at baseline, $0.500 \pm 0.100 \mathrm{pmol} / \mathrm{l}$ (mean \pm s.E.M.) and serum RANKL at month $1,0.750 \pm 0.100 \mathrm{pmol} / \mathrm{l}$. On the basis of these assumptions, we needed to enroll 20 women for the study to have a power of $80 \%$ with a type I error rate of 0.05 to detect a $0.250 \mathrm{pmol} / \mathrm{l}$ absolute difference in serum RANKL levels at different time points.

Data are presented as mean \pm s.E.M. Study parameters were of normal distribution or transformed, as revealed by the Kolmogorov-Smirnov test. The general linear model (repeated measures) was used to identify differences within the group, with Bonferroni as post hoc test. Pearson's test was used for correlation between variables. $P<0.05$ was considered statistically significant. Study power was calculated with GPower 3 (Universität Kiel, Germany). Statistical analysis was 
performed with SPSS 15 for Windows (SPSS Inc., Chicago, Illinois, USA).

\section{Results}

Serum total $\mathrm{Ca}$ increased at hour 1 after the first injection, returned to baseline values on day 1 , and was significantly higher at month 1 when compared with baseline levels. Serum P and ALP gradually increased throughout the study, reaching statistical significance at month 1. Serum P1NP was also significantly higher at month 1 when compared with baseline levels. iPTH acutely decreased by $19.5 \%$ already at hour 1 after the first injection and the reduction reached $32.6 \%$ at month 1 . Serum RANKL increased rapidly after the first injection and continued increasing towards month 1 . On the contrary, serum OPG levels remained unchanged throughout the study. OPG/RANKL ratio decreased significantly as a consequence of the significant increase in serum RANKL. All acute (up to 1 month) changes in total Ca, P, ALP, P1NP, iPTH, OPG, RANKL, and OPG/RANKL ratio are given in Table 2. Mean percent changes of serum OPG and RANKL throughout the study are illustrated in Fig. 1.

Lumbar spine BMD increased significantly in TPTDtreated women during the first year of treatment (BMD at baseline: $0.758 \pm 0.034 \mathrm{~g} / \mathrm{cm}^{2}$ versus BMD after 12 months: $0.812 \pm 0.031 \mathrm{~g} / \mathrm{cm}^{2}, P<0.001$ (increased by 7.1\%); T-score at baseline: $-3.1 \pm 0.2$ versus T-score after 12 months: $-2.8 \pm 0.2, P=0.001$ ).

No correlation was observed between serum OPG or RANKL and iPTH levels and between baseline OPG or RANKL levels and age, BMI, or BMD at baseline.

\section{Discussion}

Since the development of assays for OPG and RANKL, there has been considerable interest in their use as markers of metabolic bone disease and its treatment $(17,18)$. However, there are few convincing data that demonstrate a response of serum OPG and RANKL to therapy. A potential change in the cellular response of serum OPG or RANKL to therapy may be masked by a concurrent change in the number of active osteoblasts as bone turnover is altered. In the studies where a change in OPG has been observed, measurements have often been made within a few days after initiation of treatment, before the changes in bone turnover have been established (19). Many of these studies were of a retrospective design. Therefore, we designed a prospective study, aiming to evaluate acute (up to 1 month) changes in serum levels of OPG, RANKL, and OPG/ RANKL ratio after administration of TPTD in postmenopausal women with established osteoporosis.

In our study, the changes in serum total ALP and P1NP at 1 month and BMD at 12 months were similar to that observed in larger studies $(20,21)$, confirming the action of TPTD on bone turnover, and providing evidence of patients' compliance to treatment.

iPTH levels were gradually decreasing throughout the study. Similar reduction in endogenous PTH was observed acutely after TPTD infusion in normal (22) and osteoporotic women (23). iPTH decrease can be attributed to the suppressive effect of TPTD. Two conclusions can be made from these data: i) administration of $20 \mu \mathrm{g}$ TPTD daily is sufficient enough to inhibit endogenous hormone release from the parathyroid glands and ii) the amino-terminal end of PTH is the part of the molecule that exerts the negative feedback to the parathyroid glands.

There are serious concerns regarding the use of serum OPG and RANKL as markers of anti-osteoporotic effect on bone. First, serum OPG and RANKL may not reflect the levels and activity of these cytokines in the bone microenvironment, since a small amount of locally acting cytokines leak to systemic circulation (18). Secondly, a part of serum OPG and RANKL may originate from non-skeletal sources (18). Thirdly, the specificity of current commercially available assays to detect OPG is questioned, as they have been designed to detect all forms of OPG (monomer, dimer, RANKL/OPG complex) and not exclusively the dimeric form, which is thought to be the biologically active one (24). Fourthly, serum RANKL constitutes only a small part of total

Table 2 Study variables at baseline, $1 \mathrm{~h}, 1$ day, and 1 month after initiation of treatment.

\begin{tabular}{|c|c|c|c|c|c|}
\hline & Baseline & Hour 1 & Day 1 & Month 1 & $P$ value \\
\hline $\mathrm{Ca}(\mathrm{mg} / \mathrm{dl})$ & $9.5 \pm 0.1$ & $9.7 \pm 0.1^{*}$ & $9.5 \pm 0.1^{\dagger}$ & $9.8 \pm 0.1^{*, \neq}$ & 0.015 \\
\hline $\mathrm{P}(\mathrm{mg} / \mathrm{dl})$ & $3.8 \pm 0.1$ & $3.7 \pm 0.1$ & $3.9 \pm 0.2$ & $4.1 \pm 0.1^{\dagger}$ & 0.003 \\
\hline ALP (IU/I) & $58.3 \pm 2.1$ & $58.6 \pm 2.6$ & $61.8 \pm 4.3$ & $69.1 \pm 4.1^{*, \dagger}$ & 0.011 \\
\hline P1NP (ng/ml) & $40.6 \pm 3.6$ & & & $72.7 \pm 6.4^{*}$ & $<0.001$ \\
\hline ¡PTH (pg/ml) & $69.3 \pm 3.7$ & $55.8 \pm 3.7^{*}$ & $56.1 \pm 5.5^{\star}$ & $46.7 \pm 4.2^{*, \dagger, \ddagger}$ & $<0.001$ \\
\hline OPG (pmol/li) & $15.22 \pm 0.07$ & $15.33 \pm 0.05$ & $15.35 \pm 0.02$ & $15.38 \pm 0.05$ & 0.112 \\
\hline RANKL (pmol/l) & $0.505 \pm 0.051$ & $0.889 \pm 0.096^{*}$ & $0.958 \pm 0.125^{\star}$ & $0.994 \pm 0.120^{*}$ & $<0.001$ \\
\hline OPG/RANKL ratio & $30.1 \pm 1.4$ & $17.2 \pm 0.5$ & $16.0 \pm 0.2^{*}$ & $15.5 \pm 0.4$ & 0.044 \\
\hline
\end{tabular}

Data are shown as mean \pm S.E.M. $P$ values are calculated by the general linear model (repeated measures). ${ }^{*} P<0.05$ versus baseline, ${ }^{\dagger} P<0.05$ versus hour 1 , ${ }^{\ddagger} P<0.05$ versus day 1 (Bonferroni post hoc test). Ca, calcium; $P$, phosphate; ALP, alkaline phosphate; PINP, $N$-terminal propeptide of type I collagen; iPTH, intact parathyroid hormone; OPG, osteoprotegerin; RANKL, receptor activator for nuclear factor- $\mathrm{K} B$ ligand. 


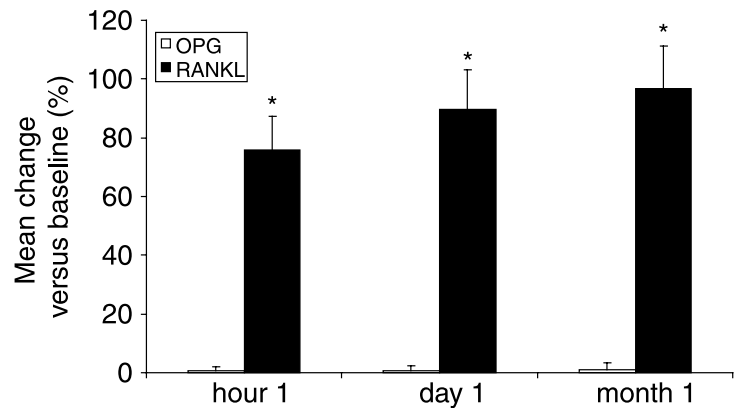

Figure 1 Changes in serum OPG and RANKL, expressed as mean percent change versus baseline, at $1 \mathrm{~h}, 1$ day, and 1 month after initiation of treatment. ${ }^{\star} P<0.05$ versus baseline (Bonferroni post hoc test).

RANKL, as the majority is cell bound and thus not detectable in the circulation. Cell surface production of RANKL can be assessed in vivo in humans by flow cytometry (25), an impractical method that usually requires bone biopsy; even if performed in peripheral blood, local (i.e., bone marrow) production of RANKL is probably the most relevant. However, in our study, serum RANKL levels, as opposed to OPG levels, changed significantly after TPTD administration.

In accordance with our results, several in vitro studies have found that PTH administration increases RANKL mRNA expression and protein levels in the osteoblasts (4-6), while it decreases (4-6) or does not affect OPG (7). Furthermore, in animal studies, an increased expression of RANKL gene following intermittent $(9,10)$ or continuous $(11,12)$ PTH administration has been reported, although OPG expression was not altered (12).

Only one study has examined TPTD effect on serum OPG and RANKL levels in humans with glucocorticoidinduced osteoporosis, but on a chronic basis (16). In this study, a significant increase in serum RANKL (within 1 month after initiation of therapy) and a mild decrease in OPG (within 6 months after initiation of therapy) were reported. Our findings are in accordance with this study, although it dealt with glucocortoid-induced osteoporosis, women were treated with $40 \mu \mathrm{g}$ TPTD daily, hormone replacement therapy was prescribed in addition to TPTD to both controls and patients, and blood samples had been subjected to two freeze-thaw cycles before analyzed with a different assay (ALPCO Diagnostics, Windham, NH, USA).

The changes in serum RANKL levels with TPTD may result from the increase in the number of active osteoblasts caused by the therapy. As the number of active osteoblasts is increasing, RANKL production increases as well, leading to the activation of the osteoclasts and the acceleration of bone turnover rate that characterizes TPTD action on the skeleton. However, extremely rapid (within $1 \mathrm{~h}$ ) changes in serum RANKL might be the result of shedding of membrane-bound RANKL, given that the increase in active osteoblasts number is not yet feasible. The anabolic effect of TPTD is possibly not exerted by the OPG/RANKL/RANK system, which regulates osteoclast activity, but by other cytokines that regulate osteoblast activity, such as insulin-like growth factor-I (IGF-I) or insulin-like growth factor-II IGF-II (26-28). IGF-I may act as a coupling factor in bone remodeling by activating both bone formation, acting directly on the osteoblasts, and bone resorption, acting indirectly on the osteoclasts through the OPG/RANKL/RANK system (26).

In conclusion, the TPTD therapy in women with postmenopausal osteoporosis results in acute increase in serum RANKL levels but does not affect serum OPG. These changes may reflect an increase in the number of active osteoblasts with therapy and might be responsible for the acceleration of bone turnover rate that characterizes TPTD.

\section{References}

1 Hodsman A, Bauer D, Dempster D, Dian L, Hanley D, Harris S, Kendler D, McClung M, Miller P, Olszynski W, Orwoll E \& Yuen C. Parathyroid hormone and teriparatide for the treatment of osteoporosis: a review of the evidence and suggested guidelines for its use. Endocrine Reviews $2005 \mathbf{2 6} 688-703$.

2 Gori F, Hofbauer L, Dunstan C, Spelsberg T, Khosla S \& Riggs B. The expression of osteoprotegerin and RANK ligand and the support of osteoclast formation by stromal-osteoblast lineage cells is developmentally regulated. Endocrinology $20001414768-4776$.

3 Hofbauer L \& Heufelder A. The role of receptor activator of nuclear factor $-\kappa \mathrm{B}$ ligand and osteoprotegerin in the pathogenesis and treatment of metabolic bone diseases. Journal of Clinical Endocrinology and Metabolism $2000 \mathbf{8 5} 2355-2363$.

4 Huang J, Sakata T, Pfleger L, Bencsik M, Halloran B, Bikle D \& Nissenson R. PTH differentially regulates expression of RANKL and OPG. Journal of Bone and Mineral Research $200419235-244$.

5 Lee S \& Lorenzo J. Parathyroid hormone stimulates TRANCE and inhibits osteoprotegerin messenger ribonucleic acid expression in murine bone marrow cultures: correlation with osteoclast-like cell formation. Endocrinology 1999140 3552-3561.

6 Kondo H, Guo J \& Bringhurst F. Cyclic adenosine monophosphate/protein kinase A mediates parathyroid hormone/parathyroid hormone-related protein receptor regulation of osteoclastogenesis and expression of RANKL and osteoprotegerin mRNAs by marrow stromal cells. Journal of Bone and Mineral Research 2002 17 1667-1679.

7 Coetzee M, Haag M \& Kruger M. Effects of arachidonic acid, docosahexaenoic acid, prostaglandin $\mathrm{E}(2)$ and parathyroid hormone on osteoprotegerin and RANKL secretion by MC3T3-E1 osteoblast-like cells. Journal of Nutritional Biochemistry $2007 \mathbf{1 8}$ 54-63.

8 Takami M, Takahashi N, Udagawa N, Miyaura C, Suda K, Woo J, Martin T, Nagai K \& Suda T. Intracellular calcium and protein kinase $\mathrm{C}$ mediate expression of receptor activator of nuclear factor$\kappa \mathrm{B}$ ligand and osteoprotegerin in osteoblasts. Endocrinology 2000 141 4711-4719.

9 Wang L, Quarles L \& Spurney R. Unmasking the osteoinductive effects of a G-protein-coupled receptor (GPCR) kinase (GRK) inhibitor by treatment with PTH (1-34). Journal of Bone and Mineral Research $2004191661-1670$.

10 Iida-Klein A, Zhou H, Lu S, Levine L, Ducayen-Knowles M, Dempster D, Nieves J \& Lindsay R. Anabolic action of parathyroid hormone is skeletal site specific at the tissue and cellular levels in mice. Journal of Bone and Mineral Research 200217 808-816. 
11 Ma Y, Cain R, Halladay D, Yang X, Zeng Q, Miles R, Chandrasekhar S, Martin T \& Onyia J. Catabolic effects of continuous human PTH (1-38) in vivo is associated with sustained stimulation of RANKL and inhibition of osteoprotegerin and geneassociated bone formation. Endocrinology $20011424047-4054$.

12 Ueno Y, Shinki T, Nagai Y, Murayama H, Fujii K \& Suda T. In vivo administration of 1,25-dihydroxyvitamin D3 suppresses the expression of RANKL mRNA in bone of thyroparathyroidectomized rats constantly infused with PTH. Journal of Cellular Biochemistry 200390 267-277.

13 Stilgren L, Rettmer E, Eriksen E, Hegedus L, Beck-Nielsen H \& Abrahamsen B. Skeletal changes in osteoprotegerin and receptor activator of nuclear factor- $\mathrm{b}$ ligand mRNA levels in primary hyperparathyroidism: effect of parathyroidectomy and association with bone metabolism. Bone 200435 256-265.

14 Stilgren L, Hegedus L, Beck-Nielsen H \& Abrahamsen B. Osteoprotegerin levels in primary hyperparathyroidism: effect of parathyroidectomy and association with bone metabolism. Calcified Tissue International 200373 210-216.

15 Seck T, Diel I, Bismar H, Ziegler R \& Pfeilschifter J. Serum parathyroid hormone, but not menopausal status, is associated with the expression of osteoprotegerin and RANKL mRNA in human bone samples. European Journal of Endocrinology 2001145 199-205.

16 Buxton E, Yao W \& Lane N. Changes in serum receptor activator of nuclear factor- $\kappa \mathrm{B}$ ligand, osteoprotegerin, and interleukin- 6 levels in patients with glucocorticoid-induced osteoporosis treated with human parathyroid hormone (1-34). Journal of Clinical Endocrinology and Metabolism $2004893332-3336$.

17 Hofbauer L \& Schoppet M. Clinical implications of the osteoprotegerin/RANKL/RANK system for bone and vascular diseases. Journal of the American Medical Association $2004292490-495$.

18 Rogers A \& Richard E. Circulating osteoprotegerin and receptor activator for nuclear factor $\kappa \mathrm{B}$ ligand: clinical utility in metabolic bone disease assessment. Journal of Clinical Endocrinology and Metabolism 200590 6323-6331.

19 Khosla S, Atkinson E, Dunstan C \& O'Fallon W. Effect of estrogen versus testosterone on circulating osteoprotegerin and other cytokine levels in normal elderly men. Journal of Clinical Endocrinology and Metabolism 200287 1550-1554.

20 Dobnig H, Sipos A, Jiang Y, Fahrleitner-Pammer A, Ste-Marie L, Gallagher J, Pavo I, Wang J \& Eriksen E. Early changes in biochemical markers of bone formation correlate with improvements in bone structure during teriparatide therapy. Journal of Clinical Endocrinology and Metabolism $2005903970-3977$.

21 Neer R, Arnaud C, Zanchetta J, Prince R, Gaich G, Reginster J, Hodsman A, Eriksen E, Ish-Shalom S, Genant H, Wang O \& Mitlak B. Effect of parathyroid hormone (1-34) on fractures and bone mineral density in postmenopausal women with osteoporosis. New England Journal of Medicine 2001344 1434-1441.

22 Kinyamu H, Gallagher J, Petranick K \& Ryschon K. Effect of parathyroid hormone (hPTH(1-34)) infusion on serum 1,25dihydroxyvitamin D and parathyroid hormone in normal women. Journal of Bone and Mineral Research 199611 1400-1405.

23 Cosman F, Shen V, Herrington B \& Lindsay R. Response of the parathyroid gland to infusion of human parathyroid hormone(1-34) (PTH-(1-34)): demonstration of suppression of endogenous secretion using immunoradiometric intact PTH-(1-84) assay. Journal of Clinical Endocrinology and Metabolism 199173 1345-1351.

24 Chen D, Sarikaya N, Gunn H, Martin S \& Young J. ELISA methodology for detection of modified osteoprotegerin in clinical studies. Clinical Chemistry 200147 747-749.

25 Eghbali-Fatourechi G, Khosla S, Sanyal A, Boyle W, Lacey D \& Riggs B. Role of RANK ligand in mediating increased bone resorption in early postmenopausal women. Journal of Clinical Investigations $2003 \mathbf{1 1 1} 1221-1230$.

26 Rubin J, Ackert-Bicknell C, Zhu L, Fan X, Murphy T, Nanes M, Marcus R, Holloway L, Beamer W \& Rosen C. IGF-I regulates osteoprotegerin (OPG) and receptor activator of nuclear factor- $\kappa \mathrm{B}$ Ligand in vitro and OPG in vivo. Journal of Clinical Endocrinology and Metabolism 200287 4273-4279.

27 Ma Y, Zeng Q, Donley D, Ste-Marie L, Gallagher J, Dalsky G, Marcus R \& Eriksen E. Teriparatide increases bone formation in modeling and remodeling osteons and enhances IGF-II immunoreactivity in postmenopausal women with osteoporosis. Journal of Bone and Mineral Research 200621 855-864.

28 Canalis E, Giustina A \& Bilezikian J. Mechanisms of anabolic therapies for osteoporosis. New England Journal of Medicine 2007 357 905-916.

Received 13 November 2007

Accepted 29 November 2007 